\title{
LOCALLY PLURIPOLAR SETS ARE PLURIPOLAR
}

\author{
DUC-VIET VU
}

ABSTRACT. We prove that every locally pluripolar set on a compact complex manifold is pluripolar. This extends similar results in Kähler case.

\section{Classification AMS 2010: 32W20, 32U40.}

Keywords: Pluripolar set, capacity, Monge-Ampère equation, Hermitian metric.

\section{INTRODUCTION}

Pluripotential theory has been a crucial tool in complex geometry, complex dynamics as well as other fields of Mathematics. We refer to [8, 12, 20, 23] for some expositions of this theory and its applications. Among other things, locally pluripolar sets are important objects in the pluripotential theory which play the role of negligible sets as a counterpart to algebraic subvarieties in algebraic geometry, see the next section for definitions. To illustrate this comparison, we recall that locally pluripolar sets are of Hausdorff codimension at least 2 (see [22, Th. 3.13]) and their intersections with totally real submanifolds of the ambient manifold are of Lebesgue measure zero (see [27, Cor. 1.2]). We refer to [19, 26, 10, 18] for more information.

Josefson's theorem [17], which is a key result in the pluripotential theory on $\mathbb{C}^{k}$, affirms that locally pluripolar sets on $\mathbb{C}^{k}$ are in fact (globally) pluripolar. Simplified proofs of this fact were given by Bedford-Taylor [3] and Alexander-Taylor [2]. This result was generalized to the pluripolar sets on projective manifolds, compact Kähler manifolds, respectively, by Dinh-Sibony [11], Guedj-Zeriahi [14], see also Berman-Boucksom-Witt Nyström [4] for the case of manifolds equipped a big line bundle. Our main result below extends this property to pluripolar sets on every compact complex manifolds.

Theorem 1.1. Every locally pluripolar set on a compact complex manifold is pluripolar.

By the above theorem, there exist abundantly non-continuous quasi-p.s.h. functions on $X$. This is a fact which probably cannot be seen directly because unlike projective manifolds, a general compact complex manifold might have very few hypersurfaces. The key ingredients of the proof of Theorem 1.1 are the comparison (2.9) between capacities generalizing similar comparison results in [2, 14] and recent developments of the pluripotential theory for non-Kähler manifolds by Kołodziej, Dinew and Nguyen [9, 21].

Acknowledgments. The author would like to thank Ngoc Cuong Nguyen for fruitful comments. This research is supported by a postdoctoral fellowship of the Alexander von Humboldt Foundation.

Date: December 4, 2018. 


\section{Proof of Theorem 1.1}

First of all, we need to recall some basic notations from the pluripotential theory. Let $X$ be a compact complex manifold of dimension $k$. A function from $X$ to $[-\infty, \infty)$ is said to be quasi-p.s.h. if it can be written locally as the sum of a plurisubharmonic (p.s.h.) function and a smooth one. Put $\mathrm{d}^{c}:=i /(2 \pi)(\bar{\partial}-\partial)$. For a continuous real $(1,1)$-form $\eta$, a quasi-p.s.h. function $\varphi$ is said to be $\eta$-p.s.h. if $\operatorname{dd}^{c} \varphi+\eta \geq 0$. We have the following characterization of quasi-p.s.h. functions in terms of submean-type inequalities.

Lemma 2.1. Let $U$ be an open subset of $\mathbb{C}^{k}$ and $\eta$ a continuous real $(1,1)$-form on $U$. A function $\varphi: U \rightarrow[-\infty, \infty)$ is $\eta$-p.s.h. if and only if it is upper semi-continuous, not identically $-\infty$ and for every $x \in U$ and every complex line $L_{v}:=\{x+t v: t \in \mathbb{C}\}$, for some $v \in \mathbb{C}^{k}$, passing through $x$, we have

$$
\varphi(x) \leq \frac{1}{2 \pi} \int_{0}^{2 \pi} \varphi\left(x+\epsilon e^{i \theta} v\right) d \theta+\int_{0}^{\epsilon} \frac{d t}{t} \int_{\{|s| \leq t\}} \eta_{v},
$$

for every constant $\epsilon>0$ small enough, where $\eta_{v}(t)$ is the restriction of $\eta$ to $L_{v}$ which is identified with $\mathbb{C}$ via $t \longmapsto x+t v$.

Proof. Consider an $\eta$-p.s.h. function $\varphi$. We need to verify (2.1). For every positive constant $r$, let $\chi_{r}$ be a smooth multi-radial nonnegative function compactly supported on the polydisk of radius $r$ in $\mathbb{C}^{k}$ with $\int_{\mathbb{C}^{k}} \chi_{r}(x) \operatorname{vol}(x)=1$, where vol is the canonical volume form on $\mathbb{C}^{k}$. Since $\varphi$ is locally integrable, we can define the convolution

$$
\varphi^{r}(x):=\int_{\mathbb{C}^{k}} \varphi(x-y) \chi_{r}(y) \operatorname{vol}(y)
$$

which is smooth. We have $\varphi^{r} \rightarrow \varphi$ pointwise as $r \rightarrow 0$ because $\varphi$ can be written as the sum of a p.s.h. function and a smooth one. Denote by

$$
\eta^{r}(x):=\int_{\mathbb{C}^{k}} \eta(x-y) \chi_{r}(y) \operatorname{vol}(y)
$$

which converges uniformly to $\eta$ as $r \rightarrow 0$ because $\eta$ is continuous. Hence, $\operatorname{dd}^{c} \varphi+\eta \geq 0$ if and only if $\operatorname{dd}^{c} \varphi^{r}+\eta^{r} \geq 0$ for every $r$ small. Similarly, (2.1) holds if it holds for $\left(\varphi^{r}, \eta^{r}\right)$ in place of $(\varphi, \eta)$ for every small $r$. It follows that it suffices to prove (2.1) for smooth $\varphi$ and smooth $\eta$.

Hence we can assume $\varphi, \eta$ are smooth and follow standard arguments in [16]. Let $v \in \mathbb{C}^{k}$ and $x \in U$. Put $\varphi_{v}(t):=\varphi(x+t v)$. We get $\operatorname{dd}^{c} \varphi_{v}+\eta_{v} \geq 0$. The Lelong-Jensen formula for $\varphi_{v}(t)$ gives

$$
M_{\epsilon, v}-M_{\epsilon^{\prime}, v}=\int_{\epsilon^{\prime}}^{\epsilon} \frac{d t}{t} \int_{\{|s| \leq t\}} \operatorname{dd}^{c} \varphi_{v},
$$

where $\epsilon>\epsilon^{\prime}$ are positive constants and

$$
M_{s, v}:=\frac{1}{2 \pi} \int_{0}^{2 \pi} \varphi_{v}\left(\epsilon e^{i \theta}\right) d \theta
$$

for every constant $s>0$. It follows that

$$
M_{\epsilon^{\prime}, v} \leq M_{\epsilon, v}+\int_{\epsilon^{\prime}}^{\epsilon} \frac{d t}{t} \int_{\{|s| \leq t\}} \eta_{v} .
$$

Letting $\epsilon^{\prime} \rightarrow 0$ in the last inequality gives (2.1) because $\varphi_{v}$ is continuous at 0 . 
Assume now (2.1). This combined with the hypothesis that $\varphi \neq-\infty$ implies $\varphi \in L_{l o c}^{1}$. Moreover, as in the case of p.s.h. functions, since $\varphi$ is upper semi-continuous, (2.1) also tells us that $\varphi$ is strongly semi-continuous in the sense that for every Borel subset $A$ of $U$ whose complement in $U$ is of zero Lebesgue measure, we have

$$
\limsup _{y \in A \rightarrow x} \varphi(y)=\varphi(x) .
$$

Consider first the case where $\varphi \in \mathscr{C}^{2}$. Direct computations show

$$
\epsilon^{-2}\left(M_{\epsilon, v}-\varphi_{v}(0)\right) \rightarrow \pi \operatorname{dd}^{c} \varphi_{v}(0) / 2
$$

as $\epsilon \rightarrow 0$. Applying this to (2.1) gives $\operatorname{dd}^{c} \varphi_{v}(0)+\eta_{v}(0) \geq 0$. In other words, we get $\operatorname{dd}^{c} \varphi+\eta \geq 0$.

In general, let $\varphi^{r}, \eta^{r}$ be as above. Since $\varphi \in L_{l o c}^{1}, \varphi^{r} \rightarrow \varphi$ in $L_{l o c}^{1}$. We see easily that (2.1) also holds for $\left(\varphi^{r}, \eta^{r}\right)$ in place of $(\varphi, \eta)$. By the above arguments, $\operatorname{dd}^{c} \varphi^{r}+\eta^{r} \geq 0$. Letting $r \rightarrow 0$ gives $\operatorname{dd}^{c} \varphi+\eta \geq 0$.

It remains to check that $\varphi$ is the sum of a p.s.h. function and a smooth one. To this end, we only need to work locally. Thus, we can assume there is a smooth function $\psi$ on $U$ with $\mathrm{dd}^{c} \psi \geq \eta$. We deduce $\mathrm{dd}^{c} \varphi_{1} \geq 0$ for $\varphi_{1}:=\varphi+\psi$ which is also strongly semi-upper continuous in the above sense. Let $\varphi_{1}^{r}$ be the regularisation of $\varphi_{1}$ defined in the same way as $\varphi^{r}$. Notice that $\varphi_{1}^{r} \rightarrow \varphi_{1}$ in $L_{l o c}^{1}$ and $\varphi_{1}^{r}$ is p.s.h. and decreasing to some p.s.h. function $\varphi_{1}^{\prime}$. Hence, $\varphi_{1}=\varphi_{1}^{\prime}$ almost everywhere. Using this and (2.2) yield that $\varphi_{1}=\varphi_{1}^{\prime}$ everywhere. In other words, $\varphi$ is quasi-p.s.h.. This ends the proof.

The following extension result generalizes the similar property for p.s.h. functions.

Lemma 2.2. Let $U$ be an open subset in a complex manifold $Y$. Let $\eta$ be a continuous real $(1,1)$-form on $Y$. Let $\psi_{1}$ be an $\eta$-p.s.h. function on $U$ and $\psi_{2}$ an $\eta$-p.s.h function on $Y$ such that $\limsup _{y \rightarrow x} \psi_{1}(y) \leq \psi_{2}(x)$ for every $x \in \partial U$. Define $\psi:=\max \left\{\psi_{1}, \psi_{2}\right\}$ on $U$ and $\psi:=\psi_{2}$ on $Y \backslash U$. Then $\psi$ is an $\eta$-p.s.h. function.

Proof. This is a direct consequence of Lemma 2.1.

A subset $A$ of $X$ is locally pluripolar if every point $x$ in $A$ there is an open neighborhood $U_{x}$ of $x$ in $X$ and a p.s.h. function $\varphi$ on $U_{x}$ for which $A \cap U_{x} \subset\{\varphi=-\infty\}$. A subset $A$ of $X$ is pluripolar if $A \subset\{\varphi=-\infty\}$ for some quasi-p.s.h. function $\varphi$ in $X$.

For every Borel set $A^{\prime}$ in an open subset $U$ of $\mathbb{C}^{k}$, Bedford-Taylor [3] introduced the following notion of capacity of $A^{\prime}$ in $U$ :

$$
\operatorname{cap}_{\mathrm{BT}}\left(\mathrm{A}^{\prime}, \mathrm{U}\right):=\sup \left\{\int_{\mathrm{A}}\left(\operatorname{dd}^{\mathrm{c}} \varphi\right)^{\mathrm{k}}: \varphi \text { p.s.h. on } U, 0 \leq \varphi \leq 1 \text { on } \mathrm{U}\right\} .
$$

Fix, from now on, a Hermitian metric $\omega$ on $X$. For every Borel set $A \subset X$, define

$$
\operatorname{cap}_{\mathrm{BTK}}(\mathrm{A}):=\sup \left\{\int_{\mathrm{A}}\left(\mathrm{dd}^{\mathrm{c}} \varphi+\omega\right)^{\mathrm{k}}: \varphi \omega \text {-p.s.h., } 0 \leq \varphi \leq 1 \text { on } \mathrm{X}\right\} \text {. }
$$

The last capacity was introduced by Kołodziej [20] as an analogue to the local capacity cap $_{\mathrm{BT}}$ and is used to study complex Monge-Ampère equations on Hermitian manifolds, see for example [20, 9, 21, 24]. By Lemma 2.4 below, cap BTK $_{\text {(A) }}$ is always finite. It is

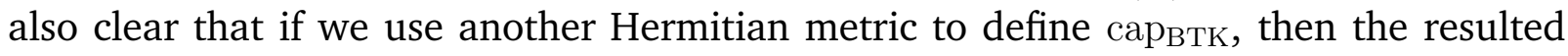
capacity is equivalent to that associated to $\omega$. 
We will need the following modified version of the classical Bedford-Taylor comparison principle due to Kołodziej and Nguyen, see [9] for a related result.

Proposition 2.3. [21, Th. 0.2] Let $\varphi, \psi$ be bounded $\omega$-p.s.h functions on $X$. Let $0<\epsilon<1$ and $m_{\epsilon}:=\inf _{X}(\varphi-(1-\epsilon) \psi)$. Then there exists a big constant $B>0$ depending only on $\omega, k$ such that for every constant $0<s<\epsilon^{3} /(16 B)$ we have

$$
\int_{\left\{\varphi<(1-\epsilon) \psi+m_{\epsilon}+s\right\}}\left((1-\epsilon) \operatorname{dd}^{c} \psi+\omega\right)^{k} \leq\left(1+C \epsilon^{-k} s\right) \int_{\left\{\varphi<(1-\epsilon) \psi+m_{\epsilon}+s\right\}}\left(\operatorname{dd}^{c} \varphi+\omega\right)^{k},
$$

where $C$ is a constant depending only on $k, B$.

A consequence of the last result is the following.

Lemma 2.4. ([9, 21]]) Let $M$ be a positive number. Then there exists a constant $c_{M}>0$ such that for every $\omega$-p.s.h. function $\varphi$ bounded by $M$, we have

$$
0<\int_{X}\left(\operatorname{dd}^{c} \varphi+\omega\right)^{k} \leq c_{M}
$$

However, we don't know whether

$$
\inf _{\{\varphi:|\varphi| \leq M\}} \int_{X}\left(\operatorname{dd}^{c} \varphi+\omega\right)^{k}>0 ?
$$

Proof. The second desired inequality is proved in [9] by using integration by parts. The first one is observed in [21]. To see it, it is enough to notice that by choosing $\epsilon:=1 / 2$ and $s>0$ small enough in Proposition 2.3, for every $\omega$-p.s.h. $\psi$ with $0 \leq \psi \leq s$ and $\varphi$ as in the hypothesis, we have

$$
\int_{\left\{\varphi<\inf _{X} \varphi+s\right\}}\left(\operatorname{dd}^{c} \psi+\omega\right)^{k} \lesssim \int_{\left\{\varphi<(1-\epsilon) \psi+m_{\epsilon}+2 s\right\}}\left((1-\epsilon) \operatorname{dd}^{c} \psi+\omega\right)^{k} \lesssim s \int_{X}\left(\operatorname{dd}^{c} \varphi+\omega\right)^{k}
$$

because

$$
\left\{\varphi<\inf _{X} \varphi+s\right\} \subset\left\{\varphi<(1-\epsilon) \psi+m_{\epsilon}+2 s\right\}
$$

It follows that

$$
\int_{X}\left(\operatorname{dd}^{c} \varphi+\omega\right)^{k} \gtrsim s \operatorname{cap}_{\text {BTK }}\left(\left\{\varphi<\inf _{\mathrm{X}} \varphi+\mathrm{s}\right\}\right)
$$

which is strictly positive because it is the capacity of a non-empty open set. The proof is finished.

Let $\left(U_{j}\right)_{1 \leq j \leq N}$ and $\left(U_{j}^{\prime}\right)_{1 \leq j \leq N}$ be finite open coverings of $X$ such that $\bar{U}_{j}$ is smooth and contained in some local chart of $X$ biholomorphic to a polydiscs for every $1 \leq j \leq N$, $U_{j}=\left\{\psi_{j}<0\right\}$ for some p.s.h. function $\psi_{j}$ defined on an open neighborhood of $\bar{U}_{j}$ with $\partial U_{j}=\left\{\psi_{j}=0\right\}$ and $U_{j}^{\prime} \Subset U_{j}$ for $1 \leq j \leq N$. In practice, it suffices to take $U_{j}, U_{j}^{\prime}$ to be balls and $\psi_{j}$ are the differences of radius functions and constants.

Lemma 2.5. ([20, 9]) There exists strictly positive constants $c_{1}, c_{2}$ such that for every $A \subset$ $X$ we have

$$
c_{1} \sum_{j=1}^{N} \operatorname{cap}_{\mathrm{BT}}\left(\mathrm{A} \cap \mathrm{U}_{\mathrm{j}}^{\prime}, \mathrm{U}_{\mathrm{j}}\right) \leq \operatorname{cap}_{\mathrm{BTK}}(\mathrm{A}) \leq \mathrm{c}_{2} \sum_{\mathrm{j}=1}^{\mathrm{N}} \operatorname{cap}_{\mathrm{BT}}\left(\mathrm{A} \cap \mathrm{U}_{\mathrm{j}}^{\prime}, \mathrm{U}_{\mathrm{j}}\right) .
$$


Proof. Put $A_{j}^{\prime}:=A \cap U_{j}^{\prime}$ which is a relatively compact subset of $U_{j}$. We have $\cup_{j} A_{j}^{\prime}=A$. The second desired inequality is obvious from the definitions of capacities. We prove now the first desired inequality.

Fix an index $1 \leq j \leq N$. By our choice of $U_{j}$, for every p.s.h. function $0 \leq u \leq 1$ on $U_{j}$, we can find another p.s.h. function $-1 \leq \tilde{u} \leq 0$ on $U_{j}$ satisfying $\tilde{u}=u-1$ on some open neighborhood of $\bar{U}_{j}^{\prime}$ and $\tilde{u}=0$ on $\partial U_{j}$. Such a $\tilde{u}$ can be chosen to be $\max \left\{u-1, A \psi_{j}\right\}$ for some constant $A$ big enough. Clearly,

$$
\int_{A_{j}^{\prime}}\left(\operatorname{dd}^{c} u\right)^{k}=\int_{A_{j}^{\prime}}\left(\operatorname{dd}^{c} \tilde{u}\right)^{k} .
$$

Since $-1 \leq \tilde{u} \leq 0$ and $\tilde{u}=0$ on $\partial U_{j}$, there is a quasi-p.s.h. function $\tilde{u}_{1}$ on $X$ such that $\mathrm{dd}^{c} \tilde{u}_{1}+C \omega \geq 0$ for some constant $C$ independent of $\tilde{u}$ and $\tilde{u}_{1}=\tilde{u}$ on some open neighborhood of $\bar{U}_{j}^{\prime}$. We deduce that

$$
\int_{A_{j}^{\prime}}\left(\operatorname{dd}^{c} u\right)^{k}=\int_{A_{j}^{\prime}}\left(\operatorname{dd}^{c} \tilde{u}_{1}\right)^{k} \leq \int_{A_{j}^{\prime}}\left(\operatorname{dd}^{c} \tilde{u}_{1}+C \omega\right)^{k} \leq C^{k} \text { сар втк }\left(\mathrm{A}_{\mathrm{j}}^{\prime}\right) .
$$

Consequently, $\operatorname{cap}_{\mathrm{BT}}\left(\mathrm{A}_{\mathrm{j}}^{\prime}, \mathrm{U}_{\mathrm{j}}\right) \leq \mathrm{C}^{\mathrm{k}} \operatorname{cap}_{\mathrm{BTK}}\left(\mathrm{A}_{\mathrm{j}}^{\prime}\right)$. Summing over $1 \leq j \leq N$ in the last inequality gives the first desired inequality. This finishes the proof.

Since we already know that if $A$ is locally pluripolar in $U$, then $\operatorname{cap}_{\text {вт }}(\mathrm{A}, \mathrm{U})=0$ (see [19, Th. 4.6.4] or [3]), we get сарвтк (A) $=0$ if $A$ is locally pluripolar in $X$. Let $\left(u_{j}\right)$ be a family of p.s.h. functions on an open subset $U$ of $\mathbb{C}^{k}$ locally bounded from above. Define $u:=\sup _{j} u_{j}$ and $u^{*}:=\sup _{j}^{*} u_{j}$ the upper semi-continuous regularisation of $u$. The set $\left\{u<u^{*}\right\}$ is called a negligible set in $U$. By Bedford-Taylor [3], the negligible sets are locally pluripolar. The following notion of capacity, which is related to those of Alexander [1] and Sibony-Wong [25], is due to Dinh-Sibony [11]: for $A \subset X$,

$$
\operatorname{cap}_{\operatorname{ADS}}(\mathrm{A}):=\inf \left\{\exp \left(\sup _{\mathrm{A}} \varphi\right): \varphi \text { w-p.s.h. on } X, \sup _{\mathrm{X}} \varphi=0\right\},
$$

see [15] for some of its applications.

Lemma 2.6. $\operatorname{cap}_{\mathrm{ADS}}(\mathrm{A})=0$ if and only if $A$ is pluripolar on $X$.

Proof. If $A \subset\{\varphi=-\infty\}$ for some quasi-p.s.h. $\varphi$, it is clear that $\operatorname{cap}_{\mathrm{ADS}}(\mathrm{A})=0$. Consider now

$$
\operatorname{cap}_{\text {ADS }}(\mathrm{A})=0 .
$$

Recall that there exists a constant $c$ such that for every $\omega$-p.s.h. function $\varphi$ with the normalization condition $\sup _{X} \varphi=0$, we have

$$
\|\varphi\|_{L^{1}(X)} \leq c .
$$

We refer to [16, 11, 9] for a proof. Using (2.5), there exists a sequence of $\omega$-p.s.h. functions $\left(\varphi_{n}\right)$ with $\sup _{X} \varphi_{n}=0$ such that $\sup _{A} \varphi_{n} \leq-n^{3}$. Put

$$
\varphi:=\sum_{n=1}^{\infty} \frac{\varphi_{n}}{n^{2}}
$$

which is a well-defined quasi-p.s.h. function because of (2.6). On the other hand,

$$
\sup _{A} \varphi \leq \sum_{n=1}^{\infty} \frac{-n^{3}}{n^{2}}=-\infty .
$$


It means that $A \subset\{\varphi=-\infty\}$. This finishes the proof.

Let $\left(\varphi_{j}\right)_{j \in J}$ be a family of $\omega$-p.s.h. functions uniformly bounded from above. Define

$$
\varphi_{J}:=\sup _{j \in J} \varphi_{j} .
$$

Observe that $\varphi_{J}^{*}$ is an $\omega$-p.s.h. function. This can be seen by using Lemma 2.1 or noticing that for every $\omega$-p.s.h. functions $\varphi_{j}, \varphi_{j^{\prime}}$ we have $\max \left\{\varphi_{j}, \varphi_{j^{\prime}}\right\}=\lim _{n \rightarrow \infty} n^{-1} \log \left(e^{n \varphi_{j}}+\right.$ $\left.e^{n \varphi_{j^{\prime}}}\right)$ whose $\mathrm{dd}^{c}$ is $\geq-\omega$ for every $n$. As in the local setting, $\left\{\varphi_{J}^{*}>\varphi_{J}\right\}$ is a locally pluripolar set. We will present below an important case of $\left(\varphi_{j}\right)_{j \in J}$ and its associated extremal function $\varphi_{J}^{*}$.

Let $A$ be a non-pluripolar subset of $X$. As in the local setting or in the Kähler case, we introduce the following extremal $\omega$-p.s.h. function:

$$
T_{A}:=\sup \{\varphi \omega \text {-p.s.h. : } \varphi \leq 0 \text { on } A\} .
$$

It is clear that $T_{A} \geq 0$. Let $T_{A}^{*}$ be the upper semi-continuous regularisation of $T_{A}$. We can check that

$$
\operatorname{cap}_{\mathrm{ADS}}(\mathrm{A})=\exp \left(-\sup _{\mathrm{x}} \mathrm{T}_{\mathrm{A}}\right) .
$$

Thus $T_{A}$ is bounded from above because $A$ is non-pluripolar. We deduce that $T_{A}^{*}$ is a bounded $\omega$-p.s.h. function and $Q_{A}:=\left\{T_{A}^{*}>T_{A}\right\}$ is a locally pluripolar set. This combined with the fact that $T_{A}=0$ on $A$ implies that $T_{A}^{*}=0$ on $A \backslash Q_{A}$. The following generalized a well-known property of $T_{A}^{*}$ in the Kähler case.

Proposition 2.7. Let $A$ be a nonpluripolar compact subset of $X$. We have

$$
\left(\mathrm{dd}^{c} T_{A}^{*}+\omega\right)^{k}=0
$$

on $X \backslash A$.

Proof. We follow the usual strategy. The key points are the existence of solutions of the Dirichlet problems proved in [21, 13, 7, 6] and Lemma 2.2 above.

By Choquet's lemma, there exists an increasing sequence of $\omega$-p.s.h. function $\varphi_{n}$ for which $T_{A}^{*}=\left(\lim _{n \rightarrow \infty} \varphi_{n}\right)^{*}$. For every $\omega$-p.s.h function $\varphi$ and every positive constant $\epsilon$, using a regularisation of $\varphi$ (see [5]), Hartog's lemma and the compactness of $A$, we deduce that there exists a smooth $\omega$-p.s.h. function $\varphi^{\prime}$ such that $\varphi \leq \varphi^{\prime}$ and $\varphi^{\prime} \leq \sup _{K} \varphi+$ $\epsilon$ on $K$. We construct a sequence $\left(\varphi_{n}^{\prime \prime}\right)$ of smooth $\omega$-p.s.h. functions from $\left(\varphi_{n}\right)$ inductively as follows. Let $\varphi_{1}^{\prime}$ be a smooth $\omega$-p.s.h. function such that $\varphi_{1} \leq \varphi_{1}^{\prime}$ and $\varphi_{1}^{\prime} \leq 1$ on $A$. For $n \geq 2$, let $\varphi_{n}^{\prime}$ be a smooth $\omega$-p.s.h. function such that

$$
\max \left\{\varphi_{n}, \varphi_{n-1}^{\prime}-(n-1)^{-2}\right\} \leq \varphi_{n}^{\prime}
$$

and $\varphi_{n}^{\prime} \leq 1 / n^{2}$ on $A$. Put

$$
\varphi_{n}^{\prime \prime}:=\varphi_{n}^{\prime}-\sum_{j=n}^{\infty} j^{-2} .
$$

By our construction, $\left(\varphi_{n}^{\prime \prime}\right)$ is increasing and $\varphi_{n}^{\prime \prime} \leq 0$ on $A$ and $\varphi_{n}^{\prime \prime} \geq \varphi_{n}-(n-1)^{-1}$ for $n \geq 2$. We infer that

$$
T_{A}^{*}=\left(\lim _{n \rightarrow \infty} \varphi_{n}^{\prime \prime}\right)^{*} .
$$

Let $\mathbb{B}$ be an open ball in $X \backslash A$. By [21, Th. 4.2], there exists $\omega$-p.s.h. functions $u_{n}$ on $\mathbb{B}$ which is in $\mathscr{C}^{0}(\overline{\mathbb{B}})$ for which $\left(\operatorname{dd}^{c} u_{n}+\omega\right)^{n}=0$ on $\mathbb{B}$ and $u_{n}=\varphi_{n}^{\prime \prime}$ on $\partial \mathbb{B}$. Define $\tilde{\varphi}_{n}^{\prime \prime}:=u_{n}$ on 
$\overline{\mathbb{B}}$ and $\tilde{\varphi}_{n}^{\prime \prime}:=\varphi_{n}^{\prime \prime}$ outside $\mathbb{B}$. By the domination principle [21, Cor. 3.4], we get $u_{n} \geq \varphi_{n}^{\prime \prime}$ on $\mathbb{B}$ and $u_{n+1} \geq u_{n}$ because $\varphi_{n+1}^{\prime \prime} \geq \varphi_{n}^{\prime \prime}$. By Lemma 2.2, $\tilde{\varphi}_{n}^{\prime \prime}$ is an $\omega$-p.s.h. function. We have obtained a sequence $\left(\tilde{\varphi}_{n}^{\prime \prime}\right)$ of continuous $\omega$-p.s.h functions increasing almost everywhere to $T_{A}^{*}$. Hence,

$$
\left(\operatorname{dd}^{c} \tilde{\varphi}_{n}^{\prime \prime}+\omega\right)^{k} \rightarrow\left(\operatorname{dd}^{c} T_{A}^{*}+\omega\right)^{k}
$$

as $n \rightarrow \infty$. We thus get $\left(\mathrm{dd}^{c} T_{A}^{*}+\omega\right)^{k}=0$ on $\mathbb{B}$ for every $\mathbb{B}$ in $X \backslash A$. The desired equality follows. This finishes the proof.

Proposition 2.8. Let $A$ be a nonpluripolar compact subset of $X$. Then there exist strictly positive constants $c_{1}, c_{2}, \lambda_{1}, \lambda_{2}$ independent of $A$ such that

$$
\exp \left(-\lambda_{1} \operatorname{cap}_{\mathrm{BTK}}^{-1}(\mathrm{~A})\right) \leq \operatorname{cap}_{\mathrm{ADS}}(\mathrm{A}) \leq \mathrm{c}_{2} \exp \left(-\lambda_{2} \mathrm{M}_{\mathrm{A}}^{1 / \mathrm{k}} \operatorname{cap}_{\mathrm{BTK}}^{-1 / \mathrm{k}}(\mathrm{A})\right) .
$$

where $M_{A}:=\int_{X}\left(\mathrm{dd}^{c} T_{A}^{*}+\omega\right)^{k}>0$.

Note that $M_{A}>0$ because of Lemma 2.4.

Proof. Since $A$ non-pluripolar, $T_{A}^{*}$ is a bounded $\omega$-p.s.h. function. By (2.7), the desired inequalities are equivalent to the following:

$$
\lambda_{1} \operatorname{cap}_{\mathrm{BTK}}^{-1}(\mathrm{~A}) \geq \sup _{\mathrm{X}} \mathrm{T}_{\mathrm{A}} \geq \mathrm{c}_{2}^{\prime}+\lambda_{2} \mathrm{M}_{\mathrm{A}}^{1 / \mathrm{k}} \operatorname{cap}_{\mathrm{BTK}}^{-1 / \mathrm{k}}(\mathrm{A})
$$

where $c_{2}^{\prime}:=-\log c_{2}$.

We prove now the first inequality of (2.10). We can assume $\sup _{X} T_{A}>0$ because otherwise the desired inequality is trivial for any $\lambda_{1} \geq 0$. Put $\varphi_{A}:=T_{A}^{*}-\sup _{X} T_{A}^{*}$ which is an $\omega$-p.s.h. function with $\sup _{X} \varphi_{A}=0$. It follows that

$$
\left\|\varphi_{A}\right\|_{L^{p}} \lesssim 1
$$

for every $p \geq 1$.

Let $\varphi$ be an $\omega$-p.s.h. function such that $0 \leq \varphi \leq 1$. Since $\left(\sup _{X} T_{A}\right)^{-1} \varphi_{A}=-1$ on $A \backslash Q_{A}$, and $\operatorname{cap}_{\mathrm{BTK}}\left(\mathrm{Q}_{\mathrm{A}}\right)=0$, we obtain

$$
\int_{A}\left(\operatorname{dd}^{c} \varphi+\omega\right)^{k} \leq\left(\sup _{X} T_{A}\right)^{-1} \int_{X}\left[-\varphi_{A}\right]\left(\operatorname{dd}^{c} \varphi+\omega\right)^{k} \lesssim\left(\sup _{X} T_{A}\right)^{-1}\left\|\varphi_{A}\right\|_{L^{1}}
$$

for every $\varphi$ with $0 \leq \varphi \leq 1$ by the Chern-Levine-Nirenberg inequality. Combining (2.12) with (2.11) gives the first inequality of (2.10). It remains to prove the second one.

Recall that $-1 \leq\left(\sup _{X} T_{A}\right)^{-1} \varphi_{A} \leq 0$ and $\left(\sup _{X} T_{A}\right)^{-1} \varphi_{A}$ is an $\left(\sup _{X} T_{A}\right)^{-1} \omega$-p.s.h. function. Hence $\left(\sup _{X} T_{A}\right)^{-1} \varphi_{A}$ is $\omega$-p.s.h. if $\left(\sup _{X} T_{A}\right)^{-1} \leq 1$. Consider the case where $\left(\sup _{X} T_{A}\right)^{-1} \leq 1$. By definition of cap $\cos _{\mathrm{BTK}}$, we get

$$
\operatorname{cap}_{\text {BTK }}(\mathrm{A}) \geq\left(\sup _{\mathrm{X}} \mathrm{T}_{\mathrm{A}}\right)^{-\mathrm{k}} \int_{\mathrm{A}}\left(\mathrm{dd}^{\mathrm{c}} \varphi_{\mathrm{A}}+\omega\right)^{\mathrm{k}}=\left(\sup _{\mathrm{X}} \mathrm{T}_{\mathrm{A}}\right)^{-\mathrm{k}} \int_{\mathrm{A}}\left(\mathrm{dd}^{\mathrm{c}} \mathrm{T}_{\mathrm{A}}^{*}+\omega\right)^{\mathrm{k}}
$$

By Proposition 2.7, we have

$$
\int_{A}\left(\operatorname{dd}^{c} T_{A}^{*}+\omega\right)^{k}=\int_{X}\left(\mathrm{dd}^{c} T_{A}^{*}+\omega\right)^{k} .
$$

Hence the second inequality of (2.10) follows if $\left(\sup _{X} T_{A}\right)^{-1} \leq 1$. When $\left(\sup _{X} T_{A}\right)^{-1} \geq 1$, then $T_{A}^{*}-1 \leq 0$ on $X$ and $\leq-1$ on $A \backslash Q_{A}$. We imply that

$$
\operatorname{cap}_{\text {BTK }}(\mathrm{A})=\operatorname{cap}_{\mathrm{BTK}}\left(\mathrm{A} \backslash \mathrm{Q}_{\mathrm{A}}\right) \geq \int_{\mathrm{A}}\left(\mathrm{dd}^{\mathrm{c}} \mathrm{T}_{\mathrm{A}}^{*}+\omega\right)^{\mathrm{k}}>0
$$


which combined with the fact that $\sup _{X} T_{A} \geq 0$ yields the second inequality of (2.10) in this case. The proof is finished.

End of the proof of Theorem 1.1. First observe that a countable union of pluripolar sets is again a pluripolar set. Indeed, let $\left(V_{n}\right)_{n \in \mathbb{N}}$ be a countable family of pluripolar sets on $X$. Hence we have $V_{n} \subset\left\{\varphi_{n}=-\infty\right\}$ for some $\omega$-p.s.h function $\varphi_{n}$ with $\sup _{X} \varphi_{n}=0$. Define

$$
\varphi:=\sum_{n=1}^{\infty} \varphi_{n} / n^{2}
$$

which is of bounded $L^{1}$-norm because $\left\|\varphi_{n}\right\|_{L^{1}}$ is uniformly bounded in $n$. Hence $\varphi$ is a quasi-p.s.h. function and $V_{n} \subset\{\varphi=-\infty\}$ for every $n$.

Let $V$ be a locally pluripolar set. We need to prove $V$ is pluripolar. If $V$ is compact, the desired claim is a direct application of (2.9). For the general case, we need some more arguments.

By Lindelöf's property, we can cover $V$ by at most countably many sets of form $\left\{\varphi_{j}=\right.$ $-\infty\}$ for some p.s.h functions $\varphi_{j}$ on some open subset $U_{j}$ of $X$. Hence in order to prove the desired assertion, we only need to consider $V=\{\varphi=-\infty\}$ for some p.s.h. function $\varphi$ in an open subset $U$ of $X$ which is biholomorphic to a ball in $\mathbb{C}^{k}$.

Let $U_{1}$ be a relatively compact open subset of $U$. Suppose that $V \cap U_{1}$ is not pluripolar. Hence $T_{V \cap U_{1}}^{*}$ is a bounded $\omega$-p.s.h function. Consider a decreasing sequence of smooth p.s.h. functions $\left(\varphi_{n}\right)_{n \in \mathbb{N}}$ defining on an open neighborhood of $\bar{U}_{1}$ converging pointwise to $\varphi$. For every positive integer $N$, put

$$
V_{n, N}:=\left\{\varphi_{n} \leq-N\right\} \cap \bar{U}_{1}
$$

which is a compact subset increasing in $n$. Hence $\left(T_{V_{n, N}}^{*}\right)_{n \in \mathbb{N}}$ is a decreasing sequence of $\omega$-p.s.h. functions which converges pointwise to an $\omega$-p.s.h. function $T_{N}$.

Since $\left\{\varphi_{n}<-N\right\}$ is open, $T_{V_{n, N}}^{*}=T_{V_{n, N}}=0$ on $\left\{\varphi_{n}<-N\right\} \cap U_{1}$. Thus $T_{N}=0$ on $\{\varphi<-N\} \cap U_{1}$ which contains $V \cap U_{1}$. We infer that

$$
0 \leq T_{N} \leq T_{V \cap U_{1}}^{*}
$$

for every $N$. This combined with the fact that $\left(T_{N}\right)_{N \in \mathbb{N}}$ is increasing gives

$$
0 \leq T_{\infty}:=\left(\lim _{N \rightarrow \infty} T_{N}\right)^{*} \leq T_{V \cap U_{1}}^{*}
$$

and $T_{\infty}$ is an $\omega$-p.s.h. function. Applying (2.9) to $A:=V_{n, N}$ we get

$$
\sup _{X} T_{V_{n, N}}^{*} \geq c_{2}^{\prime}+\lambda_{2}^{\prime} M_{n, N}^{1 / k} \operatorname{cap}_{\mathrm{BTK}}\left(\mathrm{V}_{\mathrm{n}, \mathrm{N}}\right)^{-1 / \mathrm{k}}
$$

where $M_{n, N}:=\int_{X}\left(\mathrm{dd}^{c} T_{V_{n, N}}^{*}+\omega\right)^{k}$. By the convergence of Monge-Ampère operators, we have

$$
\lim _{n \rightarrow \infty} M_{n, N}=\int_{X}\left(\operatorname{dd}^{c} T_{N}+\omega\right)^{k}=: M_{N}, \quad \lim _{N \rightarrow \infty} M_{N}=\int_{X}\left(\operatorname{dd}^{c} T_{\infty}+\omega\right)^{k}=: M_{\infty}
$$

Note that $M_{\infty}>0$ by Lemma 2.4. On the other hand, we have

$$
\operatorname{cap}_{\text {BTK }}\left(\mathrm{V}_{\mathrm{n}, \mathrm{N}}\right) \lesssim \mathrm{N}^{-1}
$$

by the Chern-Levine-Nirenberg inequality. This together with (2.16) and (2.15) implies

$$
\sup _{X} T_{N} \geq c_{2}^{\prime}+\lambda_{2}^{\prime} M_{N}^{1 / k} N^{1 / k} .
$$


Letting $N \rightarrow \infty$ in the last inequality and using (2.16), (2.14), we get

$$
\sup _{X} T_{V \cap U_{1}}^{*} \geq \sup _{X} T_{\infty}=\infty \text {. }
$$

This is a contradiction. Hence $V \cap U_{1}$ is pluripolar for every relatively compact open subset $U_{1}$ of $U$. It follows that $V$ is pluripolar. This finishes the proof.

\section{REFERENCES}

[1] H. AleXANDer, Projective capacity, in Recent developments in several complex variables (Proc. Conf., Princeton Univ., Princeton, N. J., 1979), vol. 100 of Ann. of Math. Stud., Princeton Univ. Press, Princeton, N.J., 1981, pp. 3-27.

[2] H. J. Alexander and B. A. Taylor, Comparison of two capacities in $\mathbf{C}^{n}$, Math. Z., 186 (1984), pp. 407-417.

[3] E. Bedford ANd B. A. TAYlor, A new capacity for plurisubharmonic functions, Acta Math., 149 (1982).

[4] R. Berman, S. BoucKsom, AND D. Witt NYSTRÖM, Fekete points and convergence towards equilibrium measures on complex manifolds, Acta Math., 207 (2011), pp. 1-27.

[5] Z. B£OCKI AND S. KoŁOdZIEJ, On regularization of plurisubharmonic functions on manifolds, Proc. Amer. Math. Soc., 135 (2007), pp. 2089-2093 (electronic).

[6] L. Caffarelli, J. J. Kohn, L. Nirenberg, And J. Spruck, The Dirichlet problem for nonlinear secondorder elliptic equations. II. Complex Monge-Ampère, and uniformly elliptic, equations, Comm. Pure Appl. Math., 38 (1985), pp. 209-252.

[7] P. CherRier AND A. HANANI, Le problème de Dirichlet pour les équations de Monge-Ampère en métrique hermitienne, Bull. Sci. Math., 123 (1999), pp. 577-597.

[8] J.-P. DemailLY, Complex analytic and differential geometry.http://www .fourier .ujf-grenoble.fr/ demailly.

[9] S. DineW AND S. KoŁodzIEJ, Pluripotential estimates on compact Hermitian manifolds, in Advances in geometric analysis, vol. 21 of Adv. Lect. Math. (ALM), Int. Press, Somerville, MA, 2012, pp. 69-86.

[10] T.-C. DINH, V.-A. NGUYÊN, AND N. SiBONY, Exponential estimates for plurisubharmonic functions and stochastic dynamics, J. Differential Geom., 84 (2010).

[11] T.-C. Dinh AND N. SibONY, Distribution des valeurs de transformations méromorphes et applications, Comment. Math. Helv., 81 (2006), pp. 221-258.

[12] _ Dynamics in several complex variables: endomorphisms of projective spaces and polynomial-like mappings, in Holomorphic dynamical systems, vol. 1998 of Lecture Notes in Math., Springer, Berlin, 2010, pp. 165-294.

[13] B. GuAN AND Q. LI, Complex Monge-Ampère equations and totally real submanifolds, Adv. Math., 225 (2010), pp. 1185-1223.

[14] V. GuEDJ AND A. ZERIAHI, Intrinsic capacities on compact Kähler manifolds, J. Geom. Anal., 15 (2005), pp. 607-639.

[15] F. R. HARVEY AND H. B. LAWson, JR., Projective hulls and the projective Gelfand transform, Asian J. Math., 10 (2006), pp. 607-646.

[16] L. HöRMANDER, Notions of convexity, vol. 127 of Progress in Mathematics, Birkhäuser Boston, Inc., Boston, MA, 1994.

[17] B. JosEFSON, On the equivalence between locally polar and globally polar sets for plurisubharmonic functions on $\mathbf{C}^{n}$, Ark. Mat., 16 (1978), pp. 109-115.

[18] L. Kaufmann, A Skoda-type integrability theorem for singular Monge-Ampère measures, Michigan Math. J., 66 (2017), pp. 581-594.

[19] M. KlimeK, Pluripotential theory, vol. 6 of London Mathematical Society Monographs. New Series, The Clarendon Press, Oxford University Press, New York, 1991. Oxford Science Publications.

[20] S. KoŁOdZIEJ, The complex Monge-Ampère equation and pluripotential theory, Mem. Amer. Math. Soc., 178 (2005).

[21] S. KoŁodzIEJ AND N. C. NGUYEN, Weak solutions to the complex Monge-Ampère equation on Hermitian manifolds, in Analysis, complex geometry, and mathematical physics: in honor of Duong H. Phong, vol. 644 of Contemp. Math., Amer. Math. Soc., Providence, RI, 2015, pp. 141-158. 
[22] N. S. LANDKOF, Foundations of modern potential theory, Springer-Verlag, New York-Heidelberg, 1972. Translated from the Russian by A. P. Doohovskoy, Die Grundlehren der mathematischen Wissenschaften, Band 180.

[23] N. LeVEnberg, Approximation in $\mathbb{C}^{N}$, Surv. Approx. Theory, 2 (2006), pp. 92-140.

[24] N. C. NGuYEN, The complex Monge-Ampère type equation on compact Hermitian manifolds and applications, Adv. Math., 286 (2016), pp. 240-285.

[25] N. Sibony And P. M. Wong, Some results on global analytic sets, in Séminaire Pierre Lelong-Henri Skoda (Analyse). Années 1978/79 (French), vol. 822 of Lecture Notes in Math., Springer, Berlin, 1980, pp. 221-237.

[26] H. SKODA, Sous-ensembles analytiques d'ordre fini ou infini dans $\mathbf{C}^{n}$, Bull. Soc. Math. France, 100 (1972), pp. 353-408.

[27] D.-V. VU, Complex Monge-Ampère equation for measures supported on real submanifolds, Math. Ann., 372 (2018), pp. 321-367.

University of Cologne, Mathematical Institute, Germany

E-mail address: vuduc@math. uni-koeln.de 